\title{
REVIEW
}

\section{Sexual dysfunction: A systematic review of South African research}

\author{
M M Campbell, PhD; D J Stein, FRCPC, PhD \\ Department of Psychiatry and Mental Health, Faculty of Health Sciences, University of Cape Town, South Africa
}

Corresponding author: M M Campbell (mm.campbell@uct.ac.za)

Background. The World Health Organization is in the process of revising the International Classification of Diseases and Related Health Problems (ICD). Sexual dysfunction disorders (F52) have been identified as having poor clinical utility. South Africa (SA) has been selected as one of five low- and middle-income countries in which studies will be developed to assess the clinical utility of the proposed ICD-11 revisions for sexual dysfunction disorders.

Objective. To identify scientific research generated in SA on sexual dysfunction disorders to guide these studies.

Methods. A systematic review of SA research on sexual dysfunction disorders published in peer-reviewed journals.

Results. Despite the high prevalence of ejaculatory and erectile dysfunctions, only five SA articles have addressed male sexual dysfunction since 1970. Lack of sexual interest and inability to reach orgasm are the most commonly reported complaints for women, yet only four SA articles have been published on the topic of female sexual dysfunction. Diabetes mellitus, cardiovascular disease, genitourinary disease and psychiatric or psychological disorders are common comorbid conditions associated with sexual dysfunction in both sexes, but only nine articles address sexual dysfunction as the main topic with respect to comorbid conditions.

Conclusion. Despite growing awareness of the importance of sexual health, SA-based scientific research on sexual dysfunction is limited. Further work is needed to inform recommendations for ICD-11 revisions drawn from the SA context.

S Afr Med J 2014;104(6):440-444. DOI:10.7196/SAMJ.7827

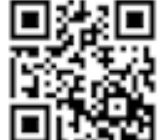

Sexual health is essential to an individual's general health, affecting quality of life and psychosocial and emotional wellbeing; however, sexual problems are estimated to affect $40-45 \%$ of women and $20-30 \%$ of men, with the prevalence increasing as individuals grow older ${ }^{[1]}$ A global study of sexual attitudes and behaviours, targeting an adult population aged $40-80$ years across 29 countries, identified early ejaculation and difficulty in achieving and maintaining an erection as the problems most commonly reported by men, affecting $24 \%$ and $17 \%$, respectively. ${ }^{[2]}$ Lack of sexual interest and inability to reach orgasm were the most commonly reported complaints for women, affecting $32 \%$ and $25 \%$, respectively. ${ }^{[2]}$ Diabetes mellitus, cardiovascular disease, genitourinary disease, psychiatric or psychological disorders, and poor general health have been identified as common comorbid conditions associated with sexual dysfunction in both sexes. ${ }^{[1]}$ However, despite the high prevalence of sexual problems and comorbidity with commonly presenting diseases and disorders, $<20 \%$ of people experiencing sexual difficulties seek assistance from healthcare providers and only $9 \%$ report being asked about their sexual health by their healthcare practitioner. $^{[1]}$

The International Classification of Diseases and Related Health Problems (ICD) is in the process of being revised by the World Health Organization (WHO), with publication of the ICD-11 planned for 2017. The WHO has identified improved clinical utility as a key goal during this revision process, with an emphasis on improving the usefulness of revised classifications for patient case conceptualisation, communication, and decision-making with respect to treatment and case management. ${ }^{[3]} \mathrm{An}$ essential element of the ICD revision process has been formative and evaluative field-testing. Formative field-testing provided feedback about the basic structure and content of the ICD and included a global survey of the opinions of 4887 psychiatrists. ${ }^{[4]}$ Results of this survey indicated that psychiatrists considered the sexual dysfunction (F52) disorders of the ICD-10 as having low ease of use and poor goodness of fit, suggesting the need for considerable revision in the ICD-11 to improve clinical utility. 
Sexual dysfunction is currently defined by the ICD-10 as 'various ways in which an individual is unable to participate in a sexual relationship as he or she would wish. There may be lack of interest, lack of enjoyment, failure of the physiological responses necessary for effective sexual interaction, or inability to control or experience orgasm. ${ }^{[5]}$ Sexual dysfunction disorders include sexual desire disorders, orgasmic dysfunction and sexual pain disorders in both sexes, as well as sexual arousal disorders in women and erectile dysfunction (ED) in men. Through an extensive review process, the WHO-appointed Working Group on the Classification of Sexual Disorders and Sexual Health has compiled suggested revisions for this category that are now ready for evaluative field-testing. However, in accordance with the recommendations made by the International Advisory Group for the Revision of the ICD-10 Mental and Behavioural Disorders, ${ }^{[3]}$ all revisions must also be considered for cultural sensitivity, relevance and appropriateness. As a result, South Africa (SA) has been selected as one of five low- and middle-income countries in which country-based field studies will be developed to assess the clinical utility of the proposed ICD-11 revisions for sexual disorders and sexual health, including sexual dysfunctions. In developing these studies, it is important to consider the scientific research on sexual dysfunction generated by SA clinicians drawing from SA population samples.

\section{Methods}

Original research articles published in English on the topic of sexual dysfunction were systematically identified using PubMed, Science Direct and EBSCOhost (including Academic Search Premier, AfricaWide Information, eBook Collection), Health Source: Nursing/ Academic Edition, MasterFILE premier, MEDLINE, PsycARTICLES, PsycINFO and SocINDEX databases. Search terms included: 'sexual dysfunct ${ }^{*}$ ' and 'South Afric ${ }^{*}$. Seventeen original research articles met the search criteria.

\section{Results}

Of the 17 articles included in this review, eight were published in international journals that included general medical journals $(n=1)$, specialist medical journals $(n=6)$ and non-medical journals $(n=1) .^{[6-13]}$ Nine were published in SA journals, eight in general medical journals (the South African Medical Journal (SAMJ) $(n=6)$ and South African Family Practice (SAFP) $(n=2))$ and one in a specialist medical journal (the South African Journal of Psychiatry $(S A J P)) .{ }^{[14-22]}$ The first article was published in the $S A M J$ in 1976, while the most recent publication appeared in SAFP in 2013. These articles are summarised in Table 1.

\section{Description of the studies}

Male sexual dysfunction was first addressed in a case series that investigated the use of clomipramine in the treatment of retrograde ejaculation following diabetic neuropathy and damage to the bladder neck. ${ }^{[14]}$ Treatment was effective in $2 / 3$ of cases.
Next a case report documented the treatment of hypogonadotrophic hypogonadism as a result of haemochromatosis, with oral testosterone. ${ }^{[9]}$

Over 15 years later, the substitution of phentolamine with chlorpromazine as an alpha-blocker in vasoactive cocktails for intracavernous injection therapy to treat $\mathrm{ED}$ was investigated. ${ }^{[15]}$ Results indicated that $96 \%(n=364)$ of patients were satisfied with the new treatment, with no significant side-effects reported.

More recently, the prevalence of anxiety and depressive symptoms in 100 men presenting for the treatment of ED was investigated using observational analysis and psychometric measures. ${ }^{[16]} \mathrm{ED}$ was severe in 57 men, moderate in 36 and mild in 7, while 60 reported premature ejaculation. Of the total sample, 41 patients were hypertensive, while 42 met the criteria for a psychiatric disorder, 33 reported depressive symptoms and 21 met the criteria for an anxiety disorder. Men suffering from ED were likely to also suffer from a psychiatric disorder and/or an accompanying general medical condition, with the probability increasing with severity of ED.

Most recently the prevalence of ED in a sample of 803 men attending a primary healthcare clinic in Durban, KwaZulu-Natal, was investigated. ${ }^{[17]}$ Results indicated that $64.9 \%(n=621)$ of the sample had ED, severe in $30.4 \%(n=244)$ of cases, moderate in $19.9 \%$ $(n=160)$, and mild in $14.6 \%(n=117)$. ED increased with age, was associated with diabetes, hypertension, cardiovascular disease and depression, and was inversely correlated with economic status.

The first SA article addressing female sexual dysfunction investigated the efficacy of a psychoeducation intervention programme for patients presenting with female orgasmic disorder at a family planning clinic. ${ }^{[18]}$ The three-stage intervention began with a history-taking session. Based on this information the patient was given a diagnosis of primary (having never experienced orgasm) or secondary (currently unable to experience orgasm) frigidity. For primary frigidity, masturbation and fantasy were recommended, while education about clitoral stimulation and different sexual positions was provided for patients with secondary frigidity. Reading material was also recommended to the couple. The second stage of intervention involved a follow-up session with the husband to elicit a medical and social history and to provide psychoeducation about the positive impact romance, dating and couple-time could have on the sexual relationship. The third and final stage involved a follow-up session with the female patient to assess whether she had achieved orgasm as a result of the intervention and to address any remaining problems. Thirty-four couples participated in the study, and 18 female patients $(53 \%)$ reported successful orgasm at termination of the intervention, 8 (24\%) reported significant sexual arousal without orgasm, and $8(24 \%)$ reported little or no arousal.

Ten years later, a case study was published on the successful treatment of a female patient (aged 25 years) presenting with vaginismus without physical cause. ${ }^{[19]}$ A psychoeducational intervention focused on reducing the female patient's anxiety about 


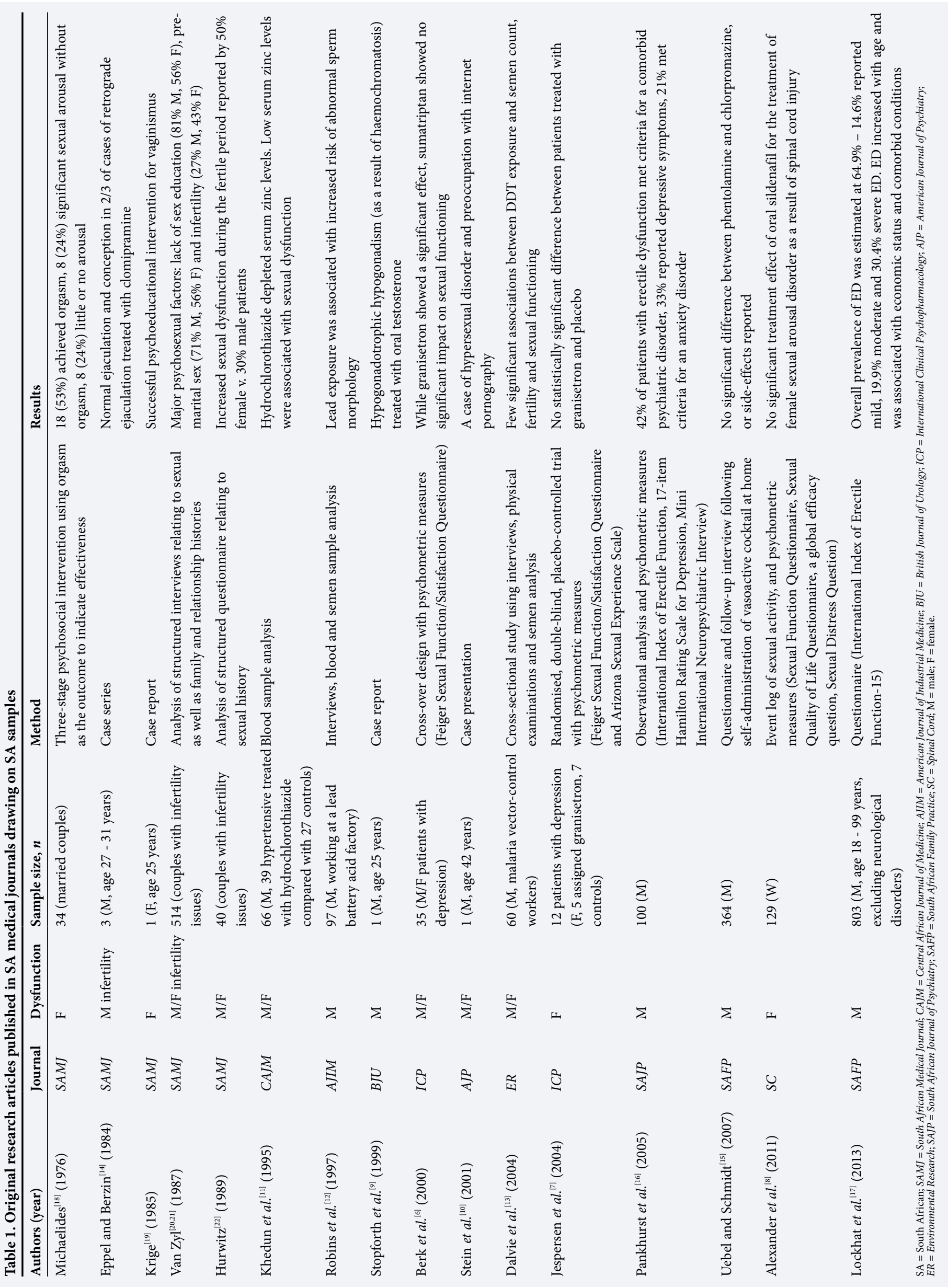


sexual penetration and gentle desensitisation through relaxation exercises, education about her genitalia, practising the use of her pubococcygeal muscles to gain a sense of control over her body, and practising penetration of her vagina with her fingers, then her husband's fingers and finally with his penis, culminating in intercourse. The joint willingness of the patient and her husband to commit to the therapy process was identified as a significant factor in the successful treatment outcome.

More recently, a pharmacotherapy trial investigated the use of granisetron to treat sexual dysfunction associated with antidepressant use in female patients diagnosed with depression. ${ }^{[7]}$ Sexual functioning was assessed at baseline, day 7 and day 14 using psychometric measures. Results indicated no significant treatment effect.

Most recently, a pharmacotherapy trial investigated the use of oral sildenafil for the treatment of female sexual arousal disorder as a result of spinal cord injury in a sample of 52 women (North America $n=23$, Europe $n=23$, Australia $n=4$, South Africa $n=2) .{ }^{[8]}$ Sexual functioning was monitored using an activity log and psychometric measures. Participants were assessed at baseline and during the 12-week treatment period. Results indicated no significant treatment effect. ${ }^{[8]}$

Studies that considered both male and female sexual dysfunction included an investigation into the psychosexual factors impacting on sexual dysfunction and their related link with infertility in a sample of 514 couples. ${ }^{[20,21]}$ Structured interviews generated information about the patients' sexual history, family background and relationship history. Analysis of responses revealed that a lack of sex education ( $81 \%$ of men and $56 \%$ of women), negative premarital sex experiences ( $70.5 \%$ of men and $56 \%$ of women) and infertility ( $27 \%$ of men and $43 \%$ of women) were the most significant psychosexual problems affecting couples presenting with infertility problems, while reduced sexual interest (lack of interest in having sex more than once a week) was reported by $68.7 \%$ of men and dyspareunia was reported by $50 \%$ of women. The study concluded that inadequate sex education and negative premarital sexual and masturbation experiences were the most significant contributors to unhelpful attitudes about sexual behaviour. The high prevalence of psychosexual problems in this SA sample highlighted the need for improved sex education for patients and improved training of medical students in sex counselling.

Shortly afterwards, a study aimed to investigate the sexual functioning of couples with primary infertility. The study investigated the influence on sexual functioning of being instructed to have sexual intercourse over the fertile period of the menstrual cycle. ${ }^{[22]}$ Couples answered questions relating to frequency of intercourse and orgasm, desire and arousal, libido, pain and sexual satisfaction, assessed on a self-rating Likert scale, across fertile and non-fertile phases of the cycle. Results indicated that $50 \%$ of female patients, as opposed to only $30 \%$ of male patients, reported increased sexual dysfunction during this fertile period, with loss of libido being the most commonly reported symptom in both sexes.

More recently, a pharmacotherapy cross-over trial investigated the use of granisetron $1 \mathrm{mg}$ and sumatriptan $100 \mathrm{mg}$ to treat sexual dysfunction associated with serotonergic antidepressant use in male and female patients diagnosed with depression. ${ }^{[6]}$ The trial included 35 patients who administered the medications 1 hour before intercourse. Sexual functioning was assessed using psychometric measures. Despite a high dropout rate, granisetron demonstrated a significant effect on sexual functioning while sumatriptan showed no significant effect.

Additional studies have included investigations of the impact of zinc, $^{[11]}$ lead ${ }^{[12]}$ and $\mathrm{DDT}^{[13]}$ on sexual functioning. Hydrochlorothiazide used for the treatment of hypertension in a sample of 39 middleaged men significantly decreased serum zinc levels. These low serum zinc levels were associated with sexual dysfunction in the sample. ${ }^{[1]}$ Lead exposure was associated with an increased risk of abnormal sperm morphology in a sample of 97 lead-exposed workers from an hSA lead battery acid factory, but did not demonstrate a significant relationship with sperm count or infertility. ${ }^{[12]}$ No strong evidence for an association between DDT exposure and semen count, fertility and sexual functioning was found in a sample of 60 malaria vectorcontrol workers in Limpopo Province. ${ }^{[13]}$

One final contribution to the SA sexual dysfunction literature is a case presentation of a 42 -year-old man presenting with a history of depression, hypersexuality and preoccupation with internet pornography, which was used to propose diagnostic criteria for hypersexual disorder. ${ }^{[10]}$

\section{Discussion}

Despite the high prevalence of ejaculatory and erectile dysfunctions reported in the international literature, ${ }^{[2]}$ only five SA articles ${ }^{[9,14-17]}$ have addressed the topic of male sexual dysfunction since 1970, with an exclusive focus on ED. Lack of sexual interest and inability to reach orgasm are the most commonly reported complaints for women ${ }^{[2]}$ yet only four SA articles ${ }^{[7,8,18,19]}$ have been published on the topic of female sexual dysfunction. Diabetes mellitus, cardiovascular disease, genitourinary disease and psychiatric or psychological disorders are common comorbid conditions associated with sexual dysfunction in both sexes; ${ }^{[1]}$ however, only nine articles address sexual dysfunction as the main topic with respect to comorbid conditions. SA research to date has focused on sexual dysfunction and diabetes, ${ }^{[14]}$ haemochromatosis, ${ }^{[9]}$ infertility, ${ }^{[20-22]}$ spinal cord injury ${ }^{[8]}$ and psychiatric conditions. ${ }^{[6,7,16,20,21]}$

Limitations of this review include that it is based only on articles published in English in peer-reviewed journals that have been electronically indexed. Nevertheless, it probably covers the most rigorous research that has been conducted in this area. 


\section{Conclusion}

SA-based scientific research on sexual dysfunction is limited and outdated, impeding credible recommendations for ICD-11 revisions drawn from the SA context. Yet SA is uniquely positioned to make a valuable contribution towards the field of sexual health. First, given its focus on human rights, SA is well placed to recognise sexual health as an important component of health. Recognition of the rights and needs of the lesbian, gay, bisexual, transgender and intersex communities in the low- and middle-income world is a key issue that continues to receive little attention in the literature. Second, sexual dysfunction demonstrates high comorbidity with HIV infection, and sub-Saharan Africa continues to experience a very high prevalence of HIV infections, with $1 / 20$ individuals infected, accounting for $69 \%$ of people living with HIV globally. ${ }^{[23]}$ Yet the impact of HIV/AIDS and other sexually transmitted infections on sexual dysfunction in the SA context remains an unexplored area, as does the influence of interpersonal violence and intimate partner violence on sexual health and functioning. Domestic violence has been identified as a considerable contributor to the burden of disease in SA, and high rates of sexual and domestic violence significantly contribute to the increased vulnerability of SA women to sexually transmitted diseases and HIV infection. ${ }^{[24]}$ However, limited SA research is currently available to guide clinicians on interventions that address both biomedical and psychosocial aspects of patients' sexual dysfunction.

The WHO-funded SA studies will provide an opportunity to generate preliminary research in this neglected area, and hopefully provide an impetus towards future research. Clinicians who wish to participate in these studies can register on the WHO Global Practice Network for sexual health practitioners (http://kuclas.qualtrics.com/SE/?SID=SV_a4SISQUBNKvVtLn).

Acknowledgements. Prof. Stein is supported by the Medical Research Council of South Africa. We thank Dr Geoffrey Reed for his inputs during the review of this manuscript.
References

1. Lewis R, Fugl-Meyer KS, Bosch R, et al. Epidemiology/risk factors of sexual dysfunction. I Sex Med 2004;1(1):35-39. [http://dx.doi.org/10.1111/j.1743-6109.2004.10106.x]

2. Moreira E, Brock G, Glasser D, et al. Help-seeking behaviour for sexual problems: The Global Study of Sexual Attitudes and Behaviors. Int J Clin Pract 2005;59(1):6-16. [http://dx.doi.org/10.1111/j.1742-1241.2005.00382.x] International Advisory Group for the Revision of ICD-10 Mental and Behavioural Disorders. A conceptua
framework for the revision of the ICD-10 classification of mental and behavioural disorders. World Psychiatry framework for the

4. Reed G, Correia J, Esparza P, Saxena S, Maj M. The WPA-WHO global survey of psychiatrists' attitudes toward mental disorders classification. World Psychiatry 2011;10(2):118-131.

5. World Health Organization. The ICD-10 Classification of Mental and Behavioural Disorders: Diagnostic Criteria for Research. Geneva: WHO, 1993. http://www.who.int/classifications/icd/en/GRNBOOK.pdf (accessed 24 March 2014)

6. Berk M, Stein DJ, Potgieter A, et al. Serotonergic targets in the treatment of antidepressant induced sexual dysfunction: a pilot study of granisetron and sumatriptan. Int Clin Psychopharmacol 2000;15(5):291-295. [http://dx.doi.org/10.1097/00004850-200015050-00006]

7. Jespersen S, Berk M, Van Wyk C, et al. A pilot randomized, double-blind, placebo-controlled study of granisetron in the treatment of sexual dysfunction in women associated with antidepressant use. Int Clin Psychopharmacol 2004;19(3):161-164. [http://dx.doi.org/10.1097/00004850-200405000-00007]

8. Alexander MS, Rosen RC, Steinberg S, Symonds T, Haughie S, Hultling C. Sildenafil in women with sexual arousal disorder following spinal cord injury. Spinal Cord 2011;49(2):273-279. [http://dx.doi.org/10.1038/ sc.2010.107]

9. Stopforth HB, Heyns CF, De Kock MLS, Sandler M. Ejaculatory failure and hypogonadotrophic hypogonadism caused by haemochromatosis. BJU Int 1999;83(6):728. [http://dx.doi.org/10.1046/j.1464-410x.1999.00064.x] 0. Stein D, Black DW, Shapira NA, Spitzer RL. Hypersexual disorder and preoccupation with interne pornography. Am J Psychiatry 2001;158(10):1590-1594. [http://dx.doi.org/10.1176/appi.ajp.158.10.1590] 1. Khedun SM, Naicker T, Maharaj B. Zinc, hydrochlorothiazide and sexual dysfunction. Cent Afr J Med 1995;41(10):312-315

12. Robins TG, Bornman MS, Ehrlich RI, et al. Semen quality and fertility of men employed in a South African lead acid battery plant. Am J Ind Med 1997;32(4):369-376. [http://dx.doi.org/10.1002/(SICI)1097-

13. Dalvie MA, Myers JE, Thompson ML, et al. The long-term effects of DDT exposure on semen, fertility and sexual function of malaria vector-control workers in Limpopo Province, South Africa. Environ Res

ing treatment of retrograde ejaculation with clomipramine hydrochloride.

15. Uebel R, Schmidt, A. The substitution of phentolamine with an equal amount of chlorpromazine as an alpha-blocker in vasoactive cocktails used for intracavernous injection therapy for the treatment of erectile dysfunction. S Afr Fam Pract 2007;49(1):14

6. Pankhurst K, Joubert G, Pretorius, P. Prevalence of anxiety and depressive symptoms in men with erectile dysfunction. South African Journal of Psychiatry 2008;11(2):57-62.

Lockhat $Y$, Ross A, Ramlachan P, Rangiah C. The prevalence of erectile dysfunction at a primary healthcare clinic in Durban, KwaZulu Natal. S Afr Fam Pract 2013;55(3):289-293.

Michaelides B. Treatment of frigidity in general practice. S Afr Med J 1976;50(34):1337-1339. [http://dx.doi. org/10.1097/00006254-197703000-00021]

20. Van Zyl J. Sex and infertility: Part I. Prevalence of psychosexual problems and subjacent factors. S Afr Med J $1987 ; 72(7): 482-484$

1. Van Zyl J. Sex and infertility: Part II. Influence of psychogenic factors and psychosexual problems. S Afr Med J 1987;72(7):485-487.

2. Hurwitz, M. Sexual dysfunction associated with infertility. A comparison of sexual function during the fertile and the non-fertile phase of the menstrual cycle. S Afr Med J 1989;76(2):58-61.

23. UNAIDS. Global Report: UNAIDS Report on the Global AIDS Epidemic 2012. Geneva: World Health Organization, 2012. http://www.unaids.org/en/media/unaids/contentassets/documents/epidemiology/2012/ gr2012/20121120_UNAIDS_Global_Report_2012_with_annexes_en.pdf (accessed 24 March 2104).

24. Dunkle K, Jewkes R. Effective HIV prevention requires gender-transformative work with men. Sex Transm Infect 2007;83(3):173-174. [http://dx.doi.org/10.1136/sti.2007.024950]

Accepted 7 January 2014. 